\title{
Impact of vaccination on epidemiology of diphtheria and pertussis
}

\author{
Guiso N* \\ Molecular Therapy and Prevention of Human Disease (URE), Institut Pasteur, Paris, France.
}

\begin{abstract}
Whooping cough and diphtheria are vaccine preventable diseases. Diphtheria, due to Corynebacterium diphtheriae or Corynebactium ulcerans, two Gram positive bacteria, is a serious upper respiratory tract disease with high morbidity and mortality rates. Vaccination, via an acellular vaccine composed only of purified, detoxified diphtheria toxin, has significantly reduced the incidence of the disease in most of the developed world. However, the lower vaccine coverage of diphtheria vaccination in adults is now a cause for concern. Furthermore, the disease remains endemic in many developing countries where the vaccine coverage is low.

Whooping cough, due to Bordetella pertussis or Bordetella parapertussis, two Gram negative bacteria, is a severe and highly contagious respiratory disease. The use of pertussis whole cell vaccines, containing inactivated bacteria, or later on pertussis acellular vaccines, only containing one to five purified, detoxified bacterial proteins, reduced dramatically the incidence of the morbidity and mortality. However, despite a high coverage the disease is still endemic in many regions of the world. Explaining this epidemiologic situation is, however, difficult since pertussis, vaccines, strategies, coverage, awareness of the disease and surveillance systems differ around the world. It is thus urgent to establish reference laboratories around the world using standardized clinical diagnosis and specific and sensitive diagnostic methods.
\end{abstract}

\section{INTRODUCTION}

Diphtheria and pertussis are two very severe respiratory diseases. They are among the oldest vaccine preventable diseases. After around eighty years of use of diphtheria acellular vaccines and pertussis whole cell vaccines and two decades of use of pertussis acellular vaccines, what are the consequences?

Diphtheria due to $C$. diphteriae is controlled in regions with a high vaccine coverage but Pertussis is not. Although intensive pertussis vaccination of young children reduced dramatically the morbidity and the mortality due to $B$. pertussis, epidemics are still occurring cyclically every 3-5 years. This review is analyzing the reasons.

\section{Consequences of Diphtheria vaccination}

Diphtheria is an acute communicable respiratory disease. The first description appeared in Hippocrates' literature. In the early $19^{\text {th }}$ century Bretonneau was the first to describe its communicability, in 1883 Klebs was the first to describe the agent of the disease and in 1884 Loeffler succeeded to culture the bacterium. In 1888, Roux and Yersin discovered the toxin produced by $C$. diphtheriae, Behring prepared the first antiserum and the first toxin-antitoxin vaccine. In the 1920s,

\footnotetext{
*Corresponding Author: Nicole Guiso, Ph.D; Molecular Therapy and Prevention of Human Diseases (URE), Institut Pasteur, 25-28 rue du Dr Roux 75724 Paris cedex 15, Paris, France.

Email: nicole.guiso@pasteur.fr
}

Ramon prepared the first toxoid by treating the toxin with formalin [1].

The illness can be respiratory or cutaneous: (i) the respiratory illness is characterized by a short incubation of one to four days followed by the development of patches of exudate that spread and become confluent. Then, a membrane covers the pharynx, the tonsils and the uvula and the cervical lymph nodes become tender. The development of the membrane can be followed by a huge inflammation and edema of the surrounding tissues associated with a higher mortality and morbidity; (ii) the cutaneous disease is a skin infection that can occur at the site of wound and may act as a source of respiratory disease [1]. .

Both respiratory and cutaneous illnesses are generally caused by $C$. diphtheriae, biotypes gravis or mitis but not belfanti; however, they can be caused by $C$. ulcerans as well. Both bacterial species possess the ability to produce the toxin because they are infected by a non-lytic phage that harbors the structural gene of the toxin in its genome. The phage integrates into specific sites in $C$. diphtheriae or $C$. ulcerans chromosome. These bacteria are called toxigenic bacteria since they produce the toxin. C. diphtheriae or C. ulcerans not carrying the phage and not producing the toxin, called non toxigenic bacteria, can also be isolated.

The disease was widespread before the introduction of vaccination and occurred cyclically every ten-twelve years. It was rare in infants less than six months presumably due to maternal antibodies and was rare in adults due to acquired immunity. After the introduction of vaccination in the 1940s, 
diphtheria declined to near elimination by 1980 from all WHO regions and is now exceptional in many of the industrialized countries. However, in the 1990s a major epidemic happened in the Russian federation with more than 150,000 cases and 5000 deaths mostly in adults [1]. The contributing factors to this epidemic included an inadequate population immunity in adolescents and adults and social conditions that facilitated the spread of the bacteria. High vaccine coverage is necessary to control the disease due to toxigenic bacteria. However, three new phenomena are observed in regions with very high vaccine coverage: (i) there are no autochthonous toxigenic $C$. diphteriae circulating. The only isolated toxigenic $C$. diphtheriae are imported and are from non-vaccinated or partially vaccinated persons [2] (ii) increase in the circulation of non-toxigenic $C$. diphtheriae. Analysis of the non-toxigenic C. diphtheriae isolated in mainland France, a highly vaccinated country, highlighted several changes. Proportion of the non- toxigenic C. diphtheriae biotype belfanti had increased between 1977 and 2013 and it is the most frequently biotype recovered in the recent years. Resistance to ciprofloxacin has increased and most isolates with decreased sensitivity belong to the belfanti biotype. Using Multi Locus Sequence Typing (MLST), we showed that $C$.diphtheria belfanti forms separate lineages [3].The non-toxigenic isolates, if lysogenized by a coryne phage, can still express diphtheria toxin. Furthermore, these non-toxigenic bacteria are able to induce disease such as respiratory, cutaneous and endocarditis or septicemia. Their surveillance is then becoming important and (iii) infection due to toxigenic $C$. ulcerans. Despite national recommendations on the use of diphtheria antitoxin and vaccination boosters, recent severe and lethal infections due to toxigenic $C$. ulcerans have been observed in France, but also in other European countries and in North America among elderly people with insufficient immunization who were in contact with cats and dogs [4,5]. We recently succeeded to prove the contamination in two cases to be due to contacts with asymptomatic straycats or an asymptomatic dog with a wound $[6,7]$. The discovery of the bacteria in pets reinforces the need to strengthen the links between animal and human health research, to better characterize the circulation of the bacteria in animals.

\section{Consequences of Pertussis vaccination}

Whooping cough, often called pertussis, is a highly contagious (R0 of 15-17) and severe respiratory disease, which is particularly life-threatening for newborns, the elderly and pregnant women [8]. De Bayou first described the disease, during an epidemic, in Paris in 1578 [9].

Clinical observations indicate that whooping cough usually lasts 6-12 weeks, sometimes longer. The disease can be divided into three main phases: (i) the catarrhal phase, which can easily be mistaken for a viral infection, lasts 7-14 days and is the period during which the individual is highly contagious; (ii) the paroxysmal phase, that can last between 2 and 8 weeks, which is the phase with the characteristic clinical symptoms including repeated episodes of coughing with whoops. Post-tussive vomiting is frequent. In addition to paroxysm, the patient may suffer apnea, bradycardia, cyanosis and bulging eyes. Paroxysms can lead to cerebral hypoxia, in turn causing seizures or encephalopathy and even sudden death; (iii) the convalescent stage is gradual and is characterized by a decrease in the frequency and severity of the cough [8].

The disease is mainly due to $B$. pertussis identified by Bordet in 1900 but was isolated by Bordet and Gengou only in 1906 [10] highlighting the difficulties in culturing the bacteria. The disease can also be due to $B$. parapertussis isolated in 1938 [11]. The disease was considered as a pediatric disease during the pre-vaccine era and occurred cyclically every 3 to 5 years [8]. Contrary to $C$. diphtheriae, difficulties in culturing $B$. pertussis hampered characterization of the virulence factors and elucidation of pathogenic mechanism of the bacteria! The first vaccine developed, was a Pertussis whole-cell vaccine (Pwv) which contains the whole inactivated $B$. pertussis organism. Vaccination of young children led to a dramatic decrease of mortality and morbidity. However, Pwv were shown (i) to be difficult to produce reproducibly, their efficacy varying from 35 to $94 \%$ [12] (ii) to induce a short duration of protection, as well as natural infection. In fact, twenty five years after the introduction of vaccination a change in the transmission of the disease was observed from a child to child transmission, to an adult to non-vaccinated infants indicating the need to introduce vaccine boosters for adolescents and adults [13]; (iii) to be reactogenic and not usable for vaccine boosters !

After decades of research, several virulence factors produced by $B$. pertussis were characterized and pertussis acellular vaccines (Pav) which includes one to five purified bacterial proteins were developed. Since the 1980s, Pav have replaced Pwv vaccines in Japan and in the 1990's in Australia, Europe, and North America. However, globally in around $60 \%$ of other countries, Pwv is still used. Both Pwv and Pav were shown to be efficacious against severe disease, provided that vaccine coverage was over $90 \%$ [12]. However, unlike diphtheria, an increase in the incidence of the disease is still cyclically reported every 3-5 years, even in areas with high vaccine coverage.

In 2012-2013 in several countries with high Pav vaccination coverage, a huge increase in pertussis case detection has been reported. Many hypotheses to explain this increase have been postulated, including increased disease awareness, improved surveillance using improved diagnostic methods such as Real Time PCR, insufficient vaccine coverage in special groups or in certain regions of large countries, vaccination not given at the recommended time, differences in vaccine-induced immunity, waning vaccine-induced immunity and the adaptability of the bacteria to protection conferred by the vaccines. The differences in vaccine induced immunity and bacterial changes recently led to several studies. In fact, it is known that the type of cellular immune response to natural infection and $\mathrm{Pw}$ vaccination is skewed to a Th1/Th17 type, whereas the response to Pav is biased towards a Th1/Th2 type. It is unclear, whether after priming with Pav, the cellular immune response to an infection would remain Th2-skewed, or it is reversed to a Th1-type [14]. Furthermore, (i) the immune protection conferred by Pav after priming with three doses seems to be slightly less efficient than the protection induced by the best Pwv which are no more on the market. However, this point is questionable as studies were performed with various methodologies and diagnostic tests. Comments about this issue have been published in the literature [15]; (ii) the duration of protection induced after vaccination with Pav, especially in older children and adolescents, seems to be shorter than that induced by natural infection or Pwv [14]. However, again epidemiological studies were performed with different methodologies and with different diagnostic tests; (iii) changes in the bacterial population of both $B$. pertussis and $B$. parapertussis species were observed in regions highly vaccinated with Pav. These changes don't impact the virulence of the bacteria but impact their fitness in $\mathrm{Pa}$ vaccinated populations [16-19]. However, no data are available in regions 
vaccinated with Pwv used in the last two decades and this is one of the major problems. Surveillance must continue to determine whether the changes in bacterial species are impacting pertussis vaccine effectiveness.

\section{CONCLUSION}

Surveillance of vaccine preventable diseases includes regular estimation of (i) vaccine coverage by public health authorities, (ii) the duration of vaccine induced protection, (iii) the effect of herd immunity on the adaptation of the agent of the disease targeted by the vaccine and (iv) Evaluation of the exact consequences of the use of vaccines under specific vaccine strategies needs to be carefully conducted using standardized clinical definition of the disease as proposed recently for pertussis [20] and use of specific and sensitive methodologies [21-22]. The understanding of the impact of vaccination, in particular pertussis vaccination, is necessary to better adapt vaccine strategies. Increase of vaccine coverage in developed countries, by introducing regular boosters throughout life, while providing effective vaccines to the rest of the world are the most prominent actions to be undertaken in the near future.

\section{REFERENCES}

1. Wharton M, Vitek CR. Diphtheria toxoid. In: Plotkin SA, Orenstein WA, eds. Vaccines, 4th ed. Philadelphia: W.B. Saunders Co. 2004;211-28.

2. Rousseau C, Belchior E, Broche B, Badell E, Guiso N, Laharie I et al. Diphtheria in the south of France, March 2011. Euro surveillance : bulletin Europeen sur les maladies transmissibles = European communicable disease bulletin. 2011;16(19).

3. Farfour E, Badell E, Dinu S, Guillot S, Guiso N. Microbiological changes and diversity in autochthonous non-toxigenic Corynebacterium diphtheriae isolated in France. Clinical microbiology and infection : the official publication of the European Society of Clinical Microbiology and Infectious Diseases. 2013;19(10):980-7. doi:10.1111/1469-0691.12103.

4. Bonmarin I, Guiso N, Le Fleche-Mateos A, Patey O, Patrick AD, LevyBruhl D. Diphtheria: a zoonotic disease in France? Vaccine. 2009;27(31):4196-200. doi:10.1016/j.vaccine.2009.04.048.

5. Wagner KS, White JM, Neal S, Crowcroft NS, Kuprevic iene N, Paberza $\mathrm{R}$, et al. Screening for Corynebacterium diphtheriae and Corynebacterium ulcerans in patients with upper respiratory tract infections 2007-2008: a multicentre European study. Clin Microbiol Infect. 2010; 17:517-25.

6. Lartigue MF, Monnet X, Le Fleche A, Grimont PA, Benet JJ, Durrbach A et al. Corynebacterium ulcerans in an immunocompromised patient with diphtheria and her dog. Journal of clinical microbiology. 2005;43(2):9991001. doi:10.1128/JCM.43.2.999-1001.2005.

7. Vandentorren S, Guiso N, Badell E, Boisrenoult P, Micaelo M, Troche G et al. Toxigenic Corynebacterium ulcerans in a fatal human case and her feline contacts, France, March 2014. Euro surveillance : bulletin Europeen sur les maladies transmissibles = European communicable disease bulletin. 2014;19(38).

8. Heininger U. Update on pertussis in children. Expert review of antiinfective therapy. 2010;8(2):163-73. doi:10.1586/eri.09.124.

9. Hegerle N, Guiso N. Epidemiology of whooping cough \& typing of Bordetella pertussis. Future microbiology. 2013;8(11):1391-403. doi:10.2217/fmb.13.111.

10. Bordet J, Gengou O. Le microbe de la coqueluche. Annales de l'Institut Pasteur 1906;20:10.

11. Eldering G, Kendrick P. Bacillus Para-Pertussis: A Species Resembling Both Bacillus Pertussis and Bacillus Bronchisepticus but Identical with Neither. Journal of bacteriology. 1938;35(6):561-72.

12. Zhang L, Prietsch SO, Axelsson I, Halperin SA. Acellular vaccines for preventing whooping cough in children. The Cochrane database of $\begin{array}{lll}\text { systematic } & \text { reviews. 2011(1):CD001478. }\end{array}$ doi:10.1002/14651858.CD001478.pub4.

13. Zepp F, Heininger U, Mertsola J, Bernatowska E, Guiso N, Roord J et al. Rationale for pertussis booster vaccination throughout life in Europe. The Lancet Infectious diseases. 2011;11(7):557-70. doi:10.1016/S14733099(11)70007-X.

14. Sheridan SL, Frith K, Snelling TL, Grimwood K, McIntyre PB, Lambert SB. Waning vaccine immunity in teenagers primed with whole cell and acellular pertussis vaccine: recent epidemiology. Expert review of vaccines. 2014;13(9):1081-106. doi:10.1586/14760584.2014.944167.

15. Guiso N. Specific biological diagnoses are needed to determine the durability of pertussis vaccine-induced immunity. Clinical infectious diseases : an official publication of the Infectious Diseases Society of America. 2012;55(10):1433-4; author reply 5-6. doi:10.1093/cid/cis671.

16. Hegerle N, Dore G, Guiso N. Pertactin deficient Bordetella pertussis present a better fitness in mice immunized with an acellular pertussis vaccine. Vaccine. 2014;32(49):6597-600. doi:10.1016/j.vaccine.2014.09.068.

17. Hegerle N, Guiso N. Bordetella pertussis and pertactin-deficient clinical isolates: lessons for pertussis vaccines. Expert review of vaccines. 2014;13(9):1135-46. doi:10.1586/14760584.2014.932254.

18. Hegerle N, Guiso N. Antibody-mediated inhibition of Bordetella pertussis adenylate cyclase-haemolysin-induced macrophage cytotoxicity is influenced by variations in the bacterial population. Microbiology. 2014;160(Pt 5):962-9. doi:10.1099/mic.0.074690-0.

19. Martin SW, Pawloski L, Williams M, Weening K, DeBolt C, Qin X et al. Pertactin-negative Bordetella pertussis strains: evidence for a possible selective advantage. Clinical infectious diseases : an official publication of the Infectious Diseases Society of America. 2015;60(2):223-7. doi: $10.1093 / \mathrm{cid} / \mathrm{ciu} 788$.

20. Cherry JD, Tan T, Wirsing von Konig CH, Forsyth KD, Thisyakorn U, Greenberg D et al. Clinical definitions of pertussis: Summary of a Global Pertussis Initiative roundtable meeting, February 2011. Clinical infectious diseases : an official publication of the Infectious Diseases Society of America. 2012;54(12):1756-64. doi:10.1093/cid/cis302.

21. WHO. Laboratory Manual for the Diagnosis of Whooping Cough caused by Bordetella pertussis/Bordetella parapertussis Update 2014. 22. Wirsing von Konig CH. Pertussis diagnostics: overview and impact of immunization. Expert Rev Vaccines 2014;13:1167-74. 